\title{
Microscopic electron models with exact SO(5) symmetry
}

\section{Citation}

Rabello, Silvio, Hiroshi Kohno, Eugene Demler, and Shou-Cheng Zhang. 1998. “Microscopic Electron Models with Exact SO(5) Symmetry." Physical Review Letters 80 (16) (April 20): 35863589. doi:10.1103/physrevlett.80.3586.

\section{Published Version}

10.1103/PhysRevLett.80.3586

\section{Permanent link}

http://nrs.harvard.edu/urn-3:HUL.InstRepos:28242407

\section{Terms of Use}

This article was downloaded from Harvard University's DASH repository, and is made available under the terms and conditions applicable to Other Posted Material, as set forth at http:// nrs.harvard.edu/urn-3:HUL.InstRepos:dash.current.terms-of-use\#LAA

\section{Share Your Story}

The Harvard community has made this article openly available.

Please share how this access benefits you. Submit a story.

Accessibility 


\title{
Microscopic Electron Models with Exact SO(5) Symmetry
}

\author{
Silvio Rabello, ${ }^{1}$ Hiroshi Kohno, ${ }^{2}$ Eugene Demler, ${ }^{1}$ and Shou-Cheng Zhang ${ }^{1}$ \\ ${ }^{1}$ Department of Physics, Stanford University, Stanford, California 94305 \\ ${ }^{2}$ Department of Physics, University of Tokyo, Bunkyo-ku, Tokyo 113, Japan
}

(Received 3 July 1997)

\begin{abstract}
We construct a class of microscopic electron models with exact $\mathrm{SO}(5)$ symmetry between antiferromagnetic and $d$-wave superconducting ground states. There is an exact one-to-one correspondence between both single-particle and collective excitations in both phases. $\mathrm{SO}(5)$ symmetry breaking terms can be introduced and classified according to irreducible representation of the exact $\mathrm{SO}(5)$ algebra. The resulting phase diagram and collective modes are identical to that of the $\mathrm{SO}(5)$ nonlinear $\sigma$ model. [S0031-9007(98)05903-1]
\end{abstract}

PACS numbers: 74.20.Mn, 71.10.-w, 74.25.Ha

One of the most interesting features of the high- $T_{c}$ superconductivity is the close proximity and the interplay between the antiferromagnetic (AF) and the $d$-wave superconducting (dSC) phases. Recently, a theoretical formalism was introduced based on the concept of a $\mathrm{SO}(5)$ symmetry between these two phases, and the resulting field-theoretical model describes the cuprate phase diagram and collective modes in a unified framework [1]. It was argued that the microscopic Hubbard model supports an approximate $\mathrm{SO}(5)$ symmetry [1-3].

In this paper, we construct a class of microscopic electron models with exact $\mathrm{SO}(5)$ symmetry. In this model, degeneracy between the AF and dSC phases can be demonstrated exactly, and both the fermionic single-particle and the bosonic collective modes can be mapped onto each other with a precise one-to-one correspondence. This model can be used as a starting point around which $\mathrm{SO}(5)$ symmetry-breaking interactions can be introduced and classified according to irreducible tensors of $\mathrm{SO}(5)$ algebra. It is shown that the resulting phase diagram and the collective modes are similar to those obtained from the effective $\mathrm{SO}(5)$ nonlinear $\sigma$ model with anisotropic couplings $[1,4]$. The purpose of this paper is to demonstrate that the general $\mathrm{SO}(5)$ idea can be realized exactly by explicit microscopic Hamiltonians. The microscopic information extracted from this class of models, especially the behavior of the fermionic excitations across the AF/dSC transition, would greatly complement the effective field theory approach. Within this class of models, we have a consistent microscopic theory of the AF/dSC transition. Since both the AF and the dSC states are stable infrared fixed points, it is plausible that one can deform the parameters so that the microscopic $\mathrm{SO}(5)$ models can also serve as a paradigm for a much more general class of $\mathrm{AF} / \mathrm{dSC}$ transitions, including those occurring in the high- $T_{c}$ cuprates and 2D organics.

The first independent attempt to construct microscopic $\mathrm{SO}(5)$ invariant models was undertaken by Henley [5]. He independently made a crucial observation that, if one replaces the standard $d$-wave factor $\cos p_{x}-\cos p_{y}$ by $\operatorname{sgn}\left(\cos p_{x}-\cos p_{y}\right)$, the $\mathrm{SO}(5)$ algebra introduced in [1] closes exactly.

It is easy to write down many electron models with exact $\mathrm{SU}(2)$ spin rotation invariance, because the electron operator $c_{\mathbf{p} \sigma}$ forms a natural spinor representation of the $\mathrm{SU}(2)$ algebra. In writing down $\mathrm{SU}(2)$ invariant models, all we have to do is to contract the spinor indices in a natural way. Therefore, the first step towards constructing a $\mathrm{SO}(5)$ invariant electron model is to find a natural definition of a $\mathrm{SO}(5)$ spinor. Spinor representations of the $\mathrm{SO}(5)$ Lie algebra can be easily constructed using the Clifford algebra of five $4 \times 4$ Dirac matrices [6] satisfying $\left\{\Gamma^{a}, \Gamma^{b}\right\}=2 \delta_{a b}(a, b=1, \ldots, 5)$, and the ten $\mathrm{SO}(5)$ rotation generators are given by $\Gamma^{a b}=-i\left[\Gamma^{a}, \Gamma^{b}\right]$. In this paper we shall use the following explicit representation for the Clifford algebra:

$$
\begin{aligned}
\Gamma^{1} & =\left(\begin{array}{cc}
0 & -i \sigma_{y} \\
i \sigma_{y} & 0
\end{array}\right) \Gamma^{(2,3,4)}=\left(\begin{array}{cc}
\vec{\sigma} & 0 \\
0 & { }^{t} \vec{\sigma}
\end{array}\right) \\
\Gamma^{5} & =\left(\begin{array}{cc}
0 & \sigma_{y} \\
\sigma_{y} & 0
\end{array}\right),
\end{aligned}
$$

where $\boldsymbol{\sigma}=\left(\sigma_{x}, \sigma_{y}, \sigma_{z}\right)$ are the usual $2 \times 2$ Pauli matrices, and the superscript $t$ means transportation. We define a four-component spinor by

$$
{ }^{t} \Psi_{\mathbf{p}}=\left\{c_{\mathbf{p} \uparrow}, c_{\mathbf{p} \downarrow}, \phi_{\pi}(\mathbf{p}) c_{-\mathbf{p}+\mathbf{Q}, \uparrow}^{\dagger}, \phi_{\pi}(\mathbf{p}) c_{-\mathbf{p}+\mathbf{Q}, \downarrow}^{\dagger}\right\},
$$

where $\phi_{\pi}(\mathbf{p})=\operatorname{sgn}\left(\cos p_{x}-\cos p_{y}\right)= \pm 1$, and $\mathbf{Q}=$ $(\pi, \pi)$. Since we have two spin degrees of freedom at a given momentum $\mathbf{p}$, such a description must be redundant. Indeed, one can easily see that the spinors with momenta outside the magnetic Brillouin zone are related to the spinors inside the magnetic Brillouin zone by an " $R$ conjugation"

$$
\Psi_{\mathbf{p}+\mathbf{Q}}=\phi_{\pi}(\mathbf{p}) R \Psi_{-\mathbf{p}}^{*} .
$$

The $R$ matrix is an invariant tensor of the $\mathrm{SO}(5)$ algebra enjoying the following properties: $R \Gamma^{a} R=-{ }^{t} \Gamma^{a}$, $R \Gamma^{a b} R={ }^{t} \Gamma^{a b}$. In our representation it takes the form $R=\left(\begin{array}{cc}0 & 1 \\ -1 & 0\end{array}\right)$. [The existence of such a matrix is related 
to the fact that the spinor representation of the $\mathrm{SO}(5) \mathrm{Lie}$ algebra is pseudoreal. The $\sigma_{y}$ matrix plays a similar role for $\mathrm{SO}(3)]$. The $\Psi_{\mathbf{p} \alpha}$ spinors obey the anticommutation relations;

$$
\begin{gathered}
\left\{\Psi_{\mathbf{p} \alpha}^{\dagger}, \Psi_{\mathbf{p}^{\prime} \beta}\right\}=\delta_{\alpha \beta} \delta_{\mathbf{p}, \mathbf{p}^{\prime}} \\
\left\{\Psi_{\mathbf{p} \alpha}^{\dagger}, \Psi_{\mathbf{p}^{\prime} \beta}\right\}=\left\{\Psi_{\mathbf{p} \alpha}, \Psi_{\mathbf{p}^{\prime} \beta}\right\}=-\phi_{\pi}(\mathbf{p}) R_{\alpha \beta} \delta_{\mathbf{p}+\mathbf{p}^{\prime}, \mathbf{Q}} .
\end{gathered}
$$

If we restrict both $\mathbf{p}$ and $\mathbf{p}^{\prime}$ inside the magnetic Brillouin zone, the right-hand side of the second equation vanishes and the $\Psi_{\mathbf{p} \alpha}$ spinors commute in the same way as the $c_{\mathbf{p} \sigma}$ spinors. They an be used to construct the $\mathrm{SO}(5)$ vector order parameter and the symmetry generators: $n_{a}=\frac{1}{4} \sum_{\mathbf{p}} w_{\mathbf{p}} \Psi_{\mathbf{p}}^{\dagger} \Gamma^{a} \Psi_{\mathbf{p}+\mathbf{Q}}$ and $L_{a b}=\frac{1}{8} \sum_{\mathbf{p}} \Psi_{\mathbf{p}}^{\dagger} \Gamma^{a b} \Psi_{\mathbf{p}}$. Here, $w_{\mathbf{p}}=w_{-\mathbf{p}}$. Note that the definition of the $\pi$ operators $\left(L_{1(2,3,4)}, L_{(2,3,4) 5}\right)$ differs from the ones used in previous works [1-3], where they are electron pair operators on the nearest-neighbor (nn) sites. The problem with this kind of definition is that the commutator algebra does not close, and generates longer-ranged bonds. Naively, the condition for the closure of the $\mathrm{SO}(5)$ algebra appears to be overconstrained. The present paper and Ref.[5] start with electron pair operators with a long-ranged profile, given in real space by the lattice Fourier transform of $\sigma_{\pi}(\mathbf{p})$,

$$
\sigma_{\pi}(m, n)=\frac{2}{\pi^{2}} \frac{1-(-)^{m+n}}{m^{2}-n^{2}},
$$

where $\mathbf{R}=(m, n)$ is a lattice point. It is truly remarkable that this simple choice closes the algebra exactly. Notice, that, while the $\pi$ operators have long-ranged profiles, the dSC order parameter can still be short ranged with suitable choices of $w_{\mathbf{p}}$. Under the $\mathrm{SO}(5)$ rotations generated by the $L_{a b}, \Psi_{\mathbf{p}}$ transforms as a proper $\mathrm{SO}(5)$ spinor

$$
\left[L_{a b}, \Psi_{\mathbf{p} \alpha}\right]=-\frac{1}{4}\left(\Gamma^{a b}\right)_{\alpha \beta} \Psi_{\mathbf{p} \beta}
$$

for all values of $\mathbf{p}$. Using these spinors, exact $\mathrm{SO}(5)$ invariant Hamiltonians can be constructed simply by proper contraction of the spinor indices.

We start with the kinetic term, and write it as

$$
H_{\mathrm{kin}}=\sum_{\mathbf{p}, \sigma} \varepsilon_{\mathbf{p}} c_{\mathbf{p}, \sigma}^{\dagger} c_{\mathbf{p}, \sigma}=\frac{1}{2} \sum_{\mathbf{p}} \varepsilon_{\mathbf{p}} \Psi_{\mathbf{p}}^{\dagger} \Psi_{\mathbf{p}} .
$$

We see that the property $\varepsilon_{\mathbf{p}+\mathbf{Q}}=-\varepsilon_{\mathbf{p}}$, valid for a nn tight binding model, is crucial for this construction to work. In order to construct four-fermion interactions, we first note that a $\mathrm{SO}(5)$ spinor bilinear can in general be decomposed into a direct sum of a scalar, a vector, and an antisymmetric tensor, i.e., $4 \times 4=1+5+10$. Therefore, general $\mathrm{SO}(5)$ invariant four-fermion interactions can be expressed as

$$
\begin{aligned}
H_{\mathrm{int}}= & \sum_{\mathbf{p}, \mathbf{p}^{\prime}, \mathbf{q}} V_{1}\left(\mathbf{p}, \mathbf{p}^{\prime} ; \mathbf{q}\right)\left(\Psi_{\mathbf{p}}^{\dagger} \Gamma^{a} \Psi_{\mathbf{p}+\mathbf{q}}\right)\left(\Psi_{\mathbf{p}^{\prime}}^{\dagger} \Gamma^{a} \Psi_{\mathbf{p}^{\prime}-\mathbf{q}}\right) \\
& +\sum_{\mathbf{p}, \mathbf{p}^{\prime}, \mathbf{q}} V_{2}\left(\mathbf{p}, \mathbf{p}^{\prime} ; \mathbf{q}\right)\left(\Psi_{\mathbf{p}}^{\dagger} \Gamma^{a b} \Psi_{\mathbf{p}+\mathbf{q}}\right)\left(\Psi_{\mathbf{p}^{\prime}}^{\dagger} \Gamma^{a b} \Psi_{\mathbf{p}^{\prime}-\mathbf{q}}\right) \\
& +\sum_{\mathbf{p}, \mathbf{p}^{\prime}, \mathbf{q}} V_{0}\left(\mathbf{p}, \mathbf{p}^{\prime} ; \mathbf{q}\right)\left(\Psi_{\mathbf{p}}^{\dagger} \Psi_{\mathbf{p}+\mathbf{q}}\right)\left(\Psi_{\mathbf{p}^{\prime}}^{\dagger} \Psi_{\mathbf{p}^{\prime}-\mathbf{q}}\right)
\end{aligned}
$$

Since $L_{a b}(\mathbf{p}, \mathbf{q}) \equiv \Psi_{\mathbf{p}}^{\dagger} \Gamma^{a b} \Psi_{\mathbf{p}+\mathbf{q}}, n_{a}(\mathbf{p}, \mathbf{q}) \equiv \Psi_{\mathbf{p}}^{\dagger} \Gamma^{a} \Psi_{\mathbf{p}+\mathbf{Q}+\mathbf{q}}$ and $\rho(\mathbf{p}, \mathbf{q}) \equiv \Psi_{\mathbf{p}}^{\dagger} \Psi_{\mathbf{p}+\mathbf{q}}$ are the true $\mathrm{SO}(5)$ tensor, vector, and scalar, respectively, for any $\mathbf{p}$ and $\mathbf{q}$; their inner products naturally gives a manifestly $\mathrm{SO}(5)$ invariant Hamiltonian.

Among three terms in $H_{\text {int }}$, we concentrate on the vector interaction (first term) in all subsequent analysis, and assume a factorizable form $V_{1}\left(\mathbf{p}, \mathbf{p}^{\prime} ; \mathbf{q}\right)=-V_{1}(\mathbf{q}) w_{\mathbf{p}} w_{\mathbf{p}^{\prime}}$. This form is not necessary, but simplifies calculations. In real space,

$$
\begin{aligned}
H_{\text {int }, 1}= & -4 \sum_{\ell, n} V_{1}\left(\mathbf{R}_{\ell}-\mathbf{R}_{n}\right) e^{i \mathbf{Q} \cdot\left(\mathbf{R}_{\ell}-\mathbf{R}_{n}\right)} \\
& \times\left[\mathbf{m}_{\ell} \cdot \mathbf{m}_{n}+\frac{1}{2}\left(\Delta_{\ell} \Delta_{n}^{\dagger}+\Delta_{\ell}^{\dagger} \Delta_{n}\right)\right] .
\end{aligned}
$$

Here, $\mathbf{m}_{\ell}$ and $\Delta_{\ell}$ are Néel and $d$-wave pairing order parameters (operators) at site $\ell \equiv \mathbf{R}_{\ell}$, but with extended internal structures determined by $w_{\mathbf{p}}$. For the simplest choice $w_{\mathbf{p}}=1$, they become

$$
\begin{gathered}
\mathbf{m}_{\ell}=\frac{1}{2}\left(\psi_{\ell}^{\dagger} \boldsymbol{\sigma} \psi_{\ell}-\chi_{\ell}^{\dagger} \boldsymbol{\sigma} \chi_{\ell}\right) e^{i \mathbf{Q} \cdot \mathbf{R}_{\ell}}, \\
\Delta_{\ell}^{\dagger}=\sum_{j} \phi_{\pi}\left(\mathbf{R}_{\ell}-\mathbf{R}_{j}\right)\left(c_{\ell \dagger}^{\dagger} c_{j \downarrow}^{\dagger}-c_{\ell \downarrow}^{\dagger} c_{j \uparrow}^{\dagger}\right) .
\end{gathered}
$$

Here, we introduced two-component spinors $\psi_{\ell}=$ ${ }^{t}\left(c_{\ell \uparrow}, c_{\ell \downarrow}\right) \quad$ and $\quad \chi_{\ell}=\left(-e^{i \mathbf{Q} \cdot \mathbf{R}_{\ell}}\right) \times{ }^{t}\left(b_{\ell \uparrow}, b_{\ell \downarrow}\right) \quad$ with $b_{\ell \sigma}=\sum_{j} \phi_{\pi}\left(\mathbf{R}_{\ell}-\mathbf{R}_{j}\right) c_{j \sigma}$. The pair wave function for dSC condensate is described by $\phi_{\pi}$ and is long ranged. For the choice $w_{\mathbf{p}}=\left|\cos p_{x}-\cos p_{y}\right|$, we obtain

$$
\begin{gathered}
\mathbf{m}_{\ell}=\frac{e^{i \mathbf{Q} \cdot \mathbf{R}}}{2} \sum_{i} \phi_{M}\left(\mathbf{R}_{\ell}-\mathbf{R}_{i}\right)\left(\psi_{i}^{\dagger} \boldsymbol{\sigma} \psi_{\ell}-\chi_{i}^{\dagger} \boldsymbol{\sigma} \chi_{\ell}\right), \\
\Delta_{\ell}^{\dagger}=\sum_{i, j} \sigma_{M}\left(\mathbf{R}_{\ell}-\mathbf{R}_{i}\right) \phi_{\pi}\left(\mathbf{R}_{\ell}-\mathbf{R}_{j}\right)\left(c_{i \uparrow}^{\dagger} c_{j \downarrow}^{\dagger}-c_{i \downarrow}^{\dagger} c_{j \dagger}^{\dagger}\right),
\end{gathered}
$$

where

$$
\phi_{M}(m, n)=\frac{4}{\pi^{2}} \frac{1+(-)^{m+n}}{\left[(m+n)^{2}-1\right]\left[(m-n)^{2}-1\right]} .
$$

The interaction between the centers of mass of $\mathbf{m}$ or $\Delta$ fields is controlled by $V_{1}(\mathbf{R})$. If we take $V_{1}(\mathbf{q})$ to be a $\delta$ function at $\mathbf{q}=\mathbf{Q}$, the $\Delta$ part in $H_{\text {int, } 1}$ becomes the usual BCS reduced Hamiltonian for $\mathrm{nn} d$-wave pairing. If 
$V_{1}(\mathbf{q})$ is taken to be a Lorenzian around $\mathbf{q}=\mathbf{Q}$, the real space form of the spin interaction resembles the potential induced by the AF paramagnon exchange [7-9].

It is not difficult to find degeneracy between AF and dSC states in the usual treatment of mean field theories. However, their excitation spectra are generally different, and quantum fluctuations may remove this degeneracy. In the $\mathrm{SO}(5)$ invariant models, symmetry not only ensures exact degeneracy of the ground states, but also ensures exact one-to-one correspondence between their excitation spectra. This fact is formulated as follows:

Theorem 1.- If $\left|\Psi_{0}\right\rangle$ is a ground state of a $\mathrm{SO}(5)$ invariant Hamiltonian with AF broken symmetry (say, in the $n_{2}$ direction), i.e., $\left\langle\Psi_{0}\left|n_{a}\right| \Psi_{0}\right\rangle=\delta_{2, a} A$, then $\left|\Psi_{0}^{\prime}\right\rangle=$ $e^{i(\pi / 2) L_{12}}\left|\Psi_{0}\right\rangle$ is a degenerate ground state with dSC broken symmetry (in $n_{1}$ direction), i.e., $\left\langle\Psi_{0}^{\prime}\left|n_{a}\right| \Psi_{0}^{\prime}\right\rangle=$ $\delta_{1, a} A$. Furthermore, all excited states of the AF ground state can be mapped to excited states of the dSC ground state at the same energy by the $e^{i(\pi / 2) L_{12}}$ operator.

The proof of this theorem is elementary, since $L_{12}$ commutes with the Hamiltonian, and $e^{-i(\pi / 2) L_{12}} n_{1} e^{i(\pi / 2) L_{12}}=$ $n_{2}$. In the following, we shall illustrate this powerful theorem in an explicit mean field calculation. We take a "generalized BCS reduced Hamiltonian" by selecting $V_{1}(\mathbf{q})=V_{1} \delta_{\mathbf{q}, \mathbf{Q}}$ in the vector interaction. The Green's function in the presence of a mean field $\left\langle n_{\mathbf{p}}^{a}\right\rangle=$ $\frac{1}{4}\left\langle\Psi_{\mathbf{p}}^{\dagger} \Gamma^{a} \Psi_{\mathbf{p}+\mathbf{Q}}\right\rangle$ is given by

$$
\begin{aligned}
G_{\alpha \beta}\left(\mathbf{p}, \mathbf{p}^{\prime} ; \omega\right) & =-i \int d t e^{i \omega t}\left\langle T \Psi_{\mathbf{p}, \alpha}(t) \Psi_{\mathbf{p}^{\prime}, \beta}^{\dagger}(0)\right\rangle \\
& =\frac{\left(\omega+\varepsilon_{\mathbf{p}}\right) \delta_{\alpha \beta} \delta_{\mathbf{p}, \mathbf{p}^{\prime}}+\Delta_{\mathbf{p}}^{a} \Gamma_{\alpha \beta}^{a} \delta_{\mathbf{p}, \mathbf{p}^{\prime}+\mathbf{Q}}}{\omega^{2}-\varepsilon_{\mathbf{p}}^{2}-\left(\Delta_{\mathbf{p}}^{a}\right)^{2}+i \delta},
\end{aligned}
$$

where $\Delta_{\mathbf{p}}^{a}=-16 V_{1} w_{\mathbf{p}} \sum_{\mathbf{k}} w_{\mathbf{k}}\left\langle n_{\mathbf{k}}^{a}\right\rangle$. This manifestly $\mathrm{SO}(5)$ invariant Green's function shows explicitly that the AF quasiparticles can be mapped onto dSC quasiparticles. In particular, the AF Green's function (in the $n_{2}$ direction) can be obtained directly from the dSC Green's function (in the $n_{1}$ direction) by a simple rotation: $G^{\mathrm{AF}}=e^{-i(\pi / 2) \Gamma_{12}} G^{\mathrm{SC}} e^{i(\pi / 2) \Gamma_{12}}$. If we take $w_{\mathbf{p}}=1$, the AF quasiparticles have a full $s$-wave gap, while the dSC quasiparticles have a full $d$-wave gap, with step discontinuity at $( \pm \pi / 2, \pm \pi / 2)$ points. For the choice of $w_{\mathbf{p}}=\left|\cos p_{x}-\cos p_{y}\right|$, the dSC quasiparticles have the usual $\cos p_{x}-\cos p_{y}$ gap behavior, while the $\mathrm{AF}$ quasiparticles have an anisotropic $s$-wave gap with nodes at $( \pm \pi / 2, \pm \pi / 2)$ points (Fig. 1). Because the AF nodes are not "topological," any small interactions will remove it [10]. In either case, the amplitude of the gaps is the same in both phases.

As symmetry-breaking perturbations to the above $\mathrm{SO}(5)$ invariant Hamiltonian, we consider two typical terms. One is the coupling to external fields $B_{a b}$,

$$
H_{\text {ext }}=-\sum_{a<b} B_{a b} L_{a b} .
$$

A particular example of this field is the chemical potential
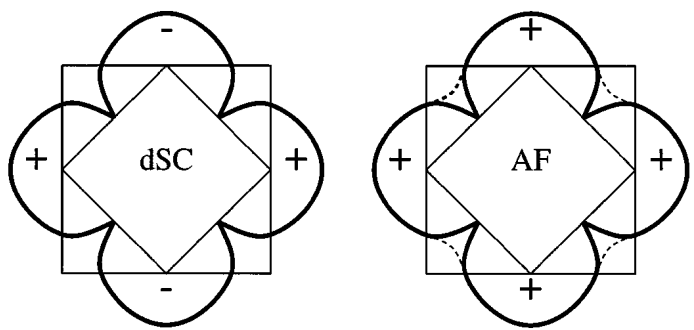

FIG. 1. The superconducting (dSC) and antiferromagnetic (AF) gaps. The solid (dotted) line in the AF gap is for the case with (without) $\mathrm{SO}(5)$ symmetry.

$B_{15}=-2 \mu$, leading to $H_{\mu}=2 \mu L_{15}$. The other is the anisotropy energy

$$
\begin{aligned}
H_{g}= & -\sum_{\mathbf{p}, \mathbf{p}^{\prime}, \mathbf{q}} \sum_{a=2,3,4} g(\mathbf{q})\left(\Psi_{\mathbf{p}}^{\dagger} \Gamma^{a} \Psi_{\mathbf{p}+\mathbf{q}}\right) \\
& \times\left(\Psi_{\mathbf{p}^{\prime}}^{\dagger} \Gamma^{a} \Psi_{\mathbf{p}^{\prime}-\mathbf{q}}\right)
\end{aligned}
$$

between AF and dSC states. To study the spectrum of $H=H_{\text {kin }}+H_{\text {int, } 1}+H_{\mu}+H_{g}$, we take $g(\mathbf{q})=g \delta_{\mathbf{q}, \mathbf{Q}}$ and again use mean field approximation. In the dSC phase, $\left\langle n_{\mathbf{p}}^{a}\right\rangle$ lies in the plane $\left(n_{1}, n_{5}\right)$. We choose it in the $n_{1}$ direction. Then the Green's function is given by

$G^{\mathrm{SC}}\left(\mathbf{p}, \mathbf{p}^{\prime}, \omega\right)=\left(\begin{array}{cc}\frac{\left(\omega+\varepsilon_{\mathbf{p}}-\mu\right) 1 \delta_{\mathbf{p}, \mathbf{p}^{\prime}}}{\omega^{2}-\left(\varepsilon_{\mathbf{p}}-\mu\right)^{2}-\Delta_{\mathbf{p}}^{2}+i \delta} & \frac{-i \sigma_{y} \Delta_{\mathbf{p}} \delta_{\mathbf{p}, \mathbf{p}^{\prime}+\mathbf{Q}}}{\omega^{2}-\left(\varepsilon_{\mathbf{p}}+\mu\right)^{2}-\Delta_{\mathbf{p}}^{2}+i \delta} \\ \frac{i \sigma_{y} \Delta_{\mathbf{p}} \delta_{\mathbf{p}, \mathbf{p}^{\prime}+\mathbf{Q}}}{\omega^{2}-\left(\varepsilon_{\mathbf{p}}-\mu\right)^{2}-\Delta_{\mathbf{p}}^{2}+i \delta} & \frac{\left(\omega+\varepsilon_{\mathbf{p}}+\mu\right) \delta_{\mathbf{p}, \mathbf{p}^{\prime}}}{\omega^{2}-\left(\varepsilon_{\mathbf{p}}+\mu\right)^{2}-\Delta_{\mathbf{p}}^{2}+i \delta}\end{array}\right)$,

where $\Delta_{\mathbf{p}}=-16 V_{1} \phi_{\pi}(\mathbf{p}) w_{\mathbf{p}} \sum_{\mathbf{k}} w_{\mathbf{k}}\left\langle n_{\mathbf{k}}^{1}\right\rangle \equiv \Delta_{0} \phi_{\pi}(\mathbf{p}) w_{\mathbf{p}}$ and the $g$ term drops out because of symmetry mismatch. $\Delta_{0}$ is determined by the gap equation $1=16 V_{1} \sum_{\mathbf{k}} \frac{w_{\mathbf{k}}^{2}}{2 E_{\mathbf{k}}}$, where $E_{\mathbf{k}}=\sqrt{\left(\varepsilon_{\mathbf{k}}-\mu\right)^{2}+\Delta_{\mathbf{k}}^{2}}$. For the choice $w_{\mathbf{p}}=$ $\left|\cos p_{x}-\cos p_{y}\right|$, we have a usual $d$-wave gap. In the $\mathrm{AF}$ phase, $\left\langle n_{\mathbf{p}}^{a}\right\rangle$ lies in the $\left(n_{2}, n_{3}, n_{4}\right)$ space. If we pick the $n_{4}$ direction, we have

$$
\begin{aligned}
& G^{\mathrm{AF}}\left(\mathbf{p}, \mathbf{p}^{\prime}, \omega\right) \\
& =\left(\begin{array}{cc}
\frac{\left(\omega_{+}+\varepsilon_{\mathbf{p}}\right) 1 \delta_{\mathbf{p}, \mathbf{p}^{\prime}}+\Delta_{\mathbf{p}} \sigma_{z} \delta_{\mathbf{p}, \mathbf{p}^{\prime}+\mathbf{Q}}}{\omega_{+}^{2}-\varepsilon_{\mathbf{p}}^{2}-\Delta_{\mathbf{p}}^{2}+i \delta} & 0 \\
0 & \frac{\left(\omega_{-}+\varepsilon_{\mathbf{p}}\right) 1 \delta_{\mathbf{p}, \mathbf{p}^{\prime}}+\Delta_{\mathbf{p}} \sigma_{z} \delta_{\mathbf{p}, \mathbf{p}^{\prime}+\mathbf{Q}}}{\omega_{-}^{2}-\varepsilon_{\mathbf{p}}^{2}-\Delta_{\mathbf{p}}^{2}+i \delta}
\end{array}\right) .
\end{aligned}
$$

Here $\omega_{ \pm}=\omega \pm \mu$ and $\Delta_{\mathbf{p}}=-16 \sum_{\mathbf{k}}\left(V_{1} w_{\mathbf{p}} w_{\mathbf{k}}+\right.$ $g)\left\langle n_{\mathbf{k}}^{4}\right\rangle \equiv w_{\mathbf{p}} \Delta_{0}+\Delta_{g} . \quad \Delta_{0}$ and $\Delta_{g}$ are determined by the gap equation $\Delta_{\mathbf{p}}=16 \sum_{\mathbf{k}}\left(V_{1} w_{\mathbf{p}} w_{\mathbf{k}}+g\right) \frac{\Delta_{\mathbf{k}}}{2 E_{\mathbf{k}}}$. The real space form of $\Delta_{\mathbf{p}}$ has an on-site contribution from $\Delta_{g}$ and a long-ranged contribution proportional to $\Delta_{0} \phi_{M}(\mathbf{R})$. We see that the $g$ terms leave the dSC gap unaffected, while it removes the AF gap node (See Fig. 1). The ground state energy curves are shown in Fig. 2. The "superspin flop" transition from AF to dSC occurs at $\mu=0$ for $H_{g}=0$, while it occurs at a finite value of $\mu_{c}$ for $g \neq 0$. In this case, the $\mathrm{AF} / \mathrm{dSC}$ transition is first order, with a finite jump in hole density $x_{c}$ (See Fig. 2).

While the above pictures are based on the mean field approximation, some exact statements can be made about the $\mathrm{AF} / \mathrm{dSC}$ transitions. $\mathrm{SO}(5)$ is a rank 2 algebra, and we can choose $Q=-L_{15}$ and $S_{z}=-L_{23}$ as the members 

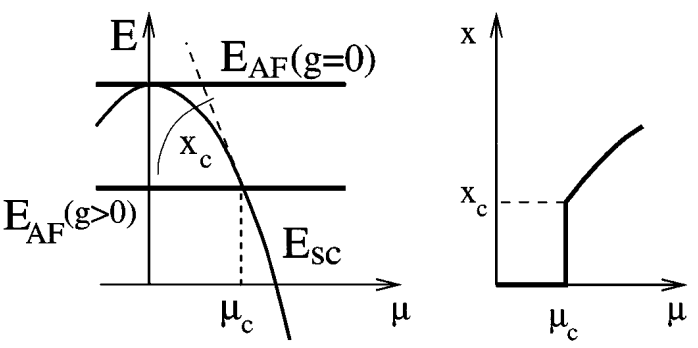

FIG. 2. The ground state energy $G$ in both AF and SC phases as functions of $\mu$ (left) and the electron density $x$ versus $\mu$ in the presence of anisotropy energy $H_{g}$ (right).

of the Cartan (maximal commutative) subalgebra. In addition, we have the Casimir operator $C=\sum_{a<b} L_{a b}^{2}$, which commutes with all of the generators and has an eigenvalue $l(l+3)$. The set $\left(Q, S_{z}, C\right)$ forms a Cartesian coordinate system labeling the quantum numbers of all states in the Hilbert space. If we consider only states with even number of electrons, these states form a pyramid, with the $l=0$ singlet on top, the $l=1$ vector next, and the $l=2$ traceless symmetric tensor on the third layer, etc. States on the same layer are all connected by the repeated actions of the eight root generators.

Theorem 2.- In $\mathrm{SO}(5)$ invariant models, it is sufficient to diagonalize the Hamiltonian at half-filling with $Q=0$ and $S_{z}=0$. All of the other states (with even number of electrons) in the Hilbert space can be obtained from these states through the action of $\left(S_{x}, S_{y}, \boldsymbol{\pi}, \boldsymbol{\pi}^{\dagger}\right)$.

In this sense, states at half-filling fully determine the states away from half-filling. In the presence of the $H_{\mu}$ term, the $\pi_{\alpha}^{\dagger}$ and $\pi_{\alpha}$ operators are exact eigenoperators of the Hamiltonian with eigenvalue $\pm 2 \mu$. Therefore, $H_{\mu}$ commutes with the Casimir operator, and simply shifts the energy of the $Q \neq 0$ states linearly without changing the wave function of these states. In a system with spontaneous symmetry breaking, lowest states with different $l$ quantum numbers are separated by an inverse system size. In the infinite system limit, these shifts, due to chemical potential, converge to the parabola as depicted in Fig. 2 [11].

The symmetry-breaking terms, $H_{g}$ and $H_{\mu}$, produce the mass gap in the Goldstone mode spectrum. For $H_{g}=0$, the mass of the $\pi$ triplet mode is exactly $2|\mu|$. For finite $H_{g}$ we employed the equations of motion (EOM) $[12,13]$ for $n^{a}$ to get the spectrum of collective modes. In the dSC state, we take $\left\langle n_{a}(\mathbf{q})\right\rangle=\left\langle n_{1}\right\rangle \delta_{a, 1} \delta_{\mathbf{q}, \mathbf{0}}$, and linearize the EOM to obtain the energy of the $\pi$ triplet $\omega_{0}=2 \sqrt{\mu^{2}-\mu_{c}^{2}}$, where $\mu_{c}=\left\langle n_{1}\right\rangle \sqrt{g V_{1}}, g \equiv g(\mathbf{Q})$, and $V_{1} \equiv V_{1}(\mathbf{Q})$, which is also consistent with the result of [1]. Similar calculation in the AF state gives the energies of the $\pi$ doublet $\omega_{0}=2\left\langle n_{4}\right\rangle \sqrt{g\left(V_{1}+g\right)} \pm 2 \mu$, where we assumed AF ordering along $n_{4}$. We therefore see that the two symmetry-breaking terms $g$ and $\mu$ partially compensate each other for the $\pi$ triplet and $Q=-2 \pi$ doublet.
In conclusion, we have constructed a class of electron models with exact $\mathrm{SO}(5)$ symmetry. If the model has an AF ground state at half-filling, it will have a dSC ground state away from half-filling. The phase diagram and the collective mode spectrum of these models are similar to the real high- $T_{c}$ materials. However, our results also show that a significant $\mathrm{SO}(5)$ symmetry-breaking term $H_{g}$ is required to produce the asymmetry in the size and angular distribution of the insulating and superconducting gaps. It is encouraging that this symmetry-breaking term transforms as a simple irreducible tensor under $\mathrm{SO}(5)$, and its consequences can be worked out systematically by the Wigner-Eckart theorem. Recent numerical calculations by Eder, Hanke, and Zhang [14] show that the two symmetrybreaking terms, $H_{g}$ and $H_{\mu}$, have compensating effects, so that a approximate $\mathrm{SO}(5)$ multiplet structure can be observed in the hole part of the spectrum in the $t-J$ model.

The authors are deeply indebted to Professor C. Henley for generous sharing of his ideas. We would like to thank Professor R. B. Laughlin for focusing our attention on the study of the AF/dSC transition problem, Professor I. Affleck, Professor E. Fradkin, Professor D. Gross, Professor M. Oshikawa, Professor J. Preskill, and Professor A. Zee for useful discussions on $\mathrm{SO}(5)$ Lie algebra, and Professor J. Berlinsky, Professor H. Fukuyama, Professor C. Kallin, Professor S. Kivelson, and Professor D. Scalapino for general discussion on the high- $T_{c}$ problem. This work is supported by the NSF under Grants No. DMR-9400372 and No. DMR-9522915. S.R. acknowledges the support from CNPq (Brazilian Research Council).

[1] S. C. Zhang, Science 275, 1089 (1997).

[2] E. Demler and S.C. Zhang, Phys. Rev. Lett. 75, 4126 (1995).

[3] S. Meixner, W. Hanke, E. Demler, and S.C. Zhang, condmat/9701217.

[4] C.P. Burgess and C. A. Lutken, cond-mat/9705216.

[5] C. Henley (unpublished).

[6] Alternatively, one can also work out the spinor representation from $\mathrm{Sp}(4)$, the universal covering group of $\mathrm{SO}(5)$.

[7] D. Scalapino, Phys. Rep. 250, 329 (1995).

[8] D. Pines, Physica (Amsterdam) 235C, 113 (1994).

[9] J. R. Schrieffer, X. G. Wen, and S.C. Zhang, Phys. Rev. B 39, 11663 (1989).

[10] P. Coleman, F.D.M. Haldane, and G. Murthy (private communication).

[11] J.C. Bonner and M.E. Fisher, Phys. Rev. 135, A640 (1964).

[12] P. W. Anderson, Phys. Rev. 112, 1900 (1958).

[13] E. Demler, S. C. Zhang, N. Bulut, and D. J. Scalapino, Int. J. Mod. Phys. B 10, 2137 (1996).

[14] R. Eder, W. Hanke, and S.C. Zhang, cond-mat/9707233. 